\section{Mitogen-activated protein kinase signaling pathways of the tangerine pathotype of Alternaria alternata}

\author{
Kuang-Ren Chung \\ Citrus Research and Education Center, \\ Institute of Food and Agricultural \\ Sciences, University of Florida, Lake \\ Alfred, FL; Department of Plant \\ Pathology, IFAS, University of Florida, \\ Gainesville, FL, USA
}

\begin{abstract}
Mitogen-activated protein kinase (MAPK)mediated signaling pathways have been known to have important functions in eukaryotic organisms. The mechanisms by which the filamentous fungus Alternaria alternata senses and responds to environmental signals have begun to be elucidated. Available data indicate that $A$. alternata utilizes the Fus $3, \operatorname{Hog} 1$ and Slt2 MAPK-mediated signaling pathways, either separately or in a cooperative manner, for conidia formation, resistance to oxidative and osmotic stress, and pathogenesis to citrus. This review provides an overview of our current knowledge of MAPK signaling pathways, in conjunction with the two-component histidine kinase and the Skn7 response regulator, in the tangerine pathotype of $A$. alternata.
\end{abstract}

\section{Introduction}

Mitogen-activated protein kinase (MAPK)mediated signaling cascades in eukaryotic microorganisms are vital for perceiving environmental stimuli at the cell surface and for transmitting these signals to the nucleus to modulate gene expression. 1,2 This signaling pathway comprises three major components: MAPK kinase kinase (MAPKKK), MAPK kinase (MAPKK), and MAPK, belonging to a family of serine/threonine protein kinases. They function in perceiving environmental stimuli by a phosphorelay mechanism.3,4 Upon perceiving environmental stimuli, MAPKKK is phosphorylated in the TXY (threonine/X/tyrosine) motif. The phosphorylated MAPKKK phosphorylates the downstream MAPKK, which in turn phosphorylates MAPK.1,5 The phosphorylated MAPK activates a number of transcription factor-coding genes, whose products eventually make a change in gene expression. Hence, microorganisms are capable of responding and adapting to their environment.

In the budding yeast Saccharomyces cere- visiae, phosphorelay systems operated by five MAPK pathways are known to regulate a wide range of cellular functions, including mating, formation of pseudo-hyphae, cell-wall reconstruction, as well as response to osmotic stress. ${ }^{6}$ Filamentous fungi have three MAPK protein kinases: the high-osmolarity glycerol (Hog1) homolog, the cell wall integrity (Slt2) homolog and the pheromone response (Fus3/Kss1) homolog.7,8 MAPK signaling pathways are well conserved among yeasts and fungi, but the biological functions of each component kinase may vary considerably in different species, largely depending on the species' lifestyles and the surrounding environment. ${ }^{9}$ Furthermore, synergistic regulations between different transduction pathways further complicate the signaling network. $6,10,11$ For example: the genes required for melanin biosynthesis and pigmentation are co-regulated by Fus3 and Slt2 MAPK-mediated pathways in the maize pathogen Cochliobolus heterostrophus. 12 In S. cerevisiae, Hogl counteracts the Fus3/Kss1 signaling pathway in response to hyperosmotic stress. ${ }^{13,14}$

Alternaria alternata is a common necrotroph, having at least ten distinct pathotypes. Each of the pathotypes produces a host-selective toxin with a distinct mode of action and induces disease only on susceptible plant species or cultivars. ${ }^{15-18}$ The tangerine pathotype of $A$. alternata produces an ACT toxin that is toxic to grapefruit ( $C$. paradisi Macfad.), tangerines ( $C$. reticulata Blanco), as well as hybrids from grapefruit and tangerine, or tangerine and sweet orange (C. sinensis (L.) 0sbeck). ${ }^{19}$ ACT, containing a 9,10-epoxy-8-hydroxy-9-methyl-decatrienoic acid structure, causes rapid electrolyte leakage from citrus cells of susceptible cultivars, and is the primary pathogenicity determinant of the tangerine pathotype of $A$. alternata. 15,16,19,20 Signaling pathways that $A$. alternata operates to respond to environmental stress have begun to be elucidated. This article summarizes the current state of knowledge of MAPK-mediated signaling pathways associated with a wide array of developmental, physiological and pathological functions in the tangerine pathotype of $A$. alternata.

\section{The Fus3/Kss1 mitogen- activated protein kinase-mediated signaling pathway in A. alternata}

The Fus3/Kss1 MAP kinases grouped in the yeast and fungal extracellular signal-regulated kinase (YERK1) subfamily5 have been intensively studied in $S$. cerevisiae. Fus3 and Kss1 are two closely related MAP kinases, functioning to regulate mating processes and/or fila-
Correspondence: Kuang-Ren Chung, University of Florida, IFAS, Citrus Research and Education Center, 700 Experiment Station Road, Lake Alfred, FL 33850, USA.

Tel. +1.863 .956 .8838 - Fax: +1.863 .956 .4631 .

E-mail krchung@ufl.edu

Key words: citrus, fungicide, osmotic, oxidative stress, phosphorylation, toxin, virulence.

Conflict of interests: the authors report no conflict of interests.

Acknowledgments: the author would like to thank current and former Chung lab members Siwy Ling Yang, Li-Hung Chen, Hsieh-Chin Tsai and Ching-Hsuan Lin for their contributions to this work. This research was supported by the Florida Agricultural Experiment Station.

Received for publication: 8 January 2013. Revision received: 27 February 2013. Accepted for publication: 27 February 2013.

This work is licensed under a Creative Commons Attribution NonCommercial 3.0 License (CC BYNC 3.0).

\section{(C)Copyright K-R. Chung, 2013}

Licensee PAGEPress, Italy

MAP Kinase 2013; 2:e4

doi:10.4081/mk.2013.e4

mentous growth. The Fus3 and Kss1 pathways in $S$. cerevisiae are partially redundant in which both share a number of components through the MAPK signaling pathway. However, Fus3 is predominately responsible for mating process, whereas Kss1 is for filamentous growth. ${ }^{21}$ In response to availability of nitrogen or pheromone, the Fus3/Kss1 MAP kinase signaling pathway is activated by the $\mathrm{G}$ protein/proteinase kinase A (PKA)-mediated signaling pathways and regulates numerous genes required for mating and/or hyphal development. Many filamentous fungi also have the Fus3 homologs. The Magnaporthe grisea PMK1 that was able to complement an $S$. cerevisiae mutant defective in mating is essential for appressorium formation and pathogenicity on rice. ${ }^{22}$ The Fus3 homolog also plays a critical role in virulence of other phytopathogenic fungi, including $A$. brassicicola, Bipolaris oryzae, Botrytis cinerea, Claviceps purpurea, $C$. heterostrophus, Colletotrichum lagenarium, Cryphonectria parasitica, Fusarium spp., Mycosphaerella graminicola, Pyrenophora teres, Stagonospora nodorum and Verticillium dahliae. ${ }^{23-37}$ The Fus3 homolog (Cek1) is also an important virulence determinant in the opportunistic human pathogen Candida albicans. 38

The AaFus3 gene encoding a homolog of the yeast Fus3-like MAP kinase was cloned and 
characterized from the tangerine pathotype of A. alternata. ${ }^{39}$ The AaFus3 gene encodes a polypeptide of 352 amino acids, which contain a protein kinase active site, a threonine/glutamic acid/tyrosine (TEY) activation motif and an ATP-binding domain, a characteristic MAP kinase motif. AaFus3 is most similar to the $P$. teres PTK1, the Bi. oryzae BMPK1, the A. brassicicola AMK1 and the Co. heterostrophus ChK1, and is less similar to the Botryotinia fuckeliana BMP1, the M. grisea PMK1 and the My. graminicola MgFus3. Systemic loss-offunction genetics in A. alternata reveal that AaFus3 regulates a number of physiological metabolisms and development in A. alternata (Figure 1). The A. alternata strain lacking AaFus3 ( $\triangle$ fus3) exhibited growth retardation. Fungal growth could be restored to wild-type levels in a strain that re-acquired a wild-type copy of AaFus3. Application of glucose, but not sorbitol, mannitol or sucrose (each at $1.5 \mathrm{M}$ ), partially restored vegetative growth of the $\Delta$ fus3 mutants. The $\Delta$ fus3 mutants grew faster than wild type or the complementation strains in the presence of potassium chloride $(\mathrm{KCl})$ or sodium chloride $(\mathrm{NaCl})$, implicating the AaFus3-mediated signaling pathway in negative regulation of salt tolerance. The AaFus3 pathway is also involved in fungicide resistance because the $\Delta$ fus 3 mutants are more sensitive to copper fungicides than the wild-type and the complementation strains. ${ }^{39}$

In addition, the Fus3 MAPK-mediated signaling pathway is involved in conidia formation and maturation in A. alternata, since $\Delta$ fus3 mutant does not produce any conidia. The $\Delta$ fus3 mutant generates dark, aberrant hyphae with distinct septae that occur in chains, but never produces fully mature conidia. Administration of cyclic adenosine monophosphate (cAMP), $\mathrm{NaCl}, \mathrm{KCl}$, yeast extracts or various antioxidants such as $\alpha$ tocopherol (vitamin E), ascorbic acid and proline could not enhance fungal growth or revive conidiation of fungal strains impaired for AaFus3. However, application of glucose restored vegetative growth, but not conidia formation. Conidia are vital for completing the $A$. alternata life cycle and for initiating disease on citrus. Similar defects in conidia maturation were also observed in A. brassicicola lacking a Fus3/Kss1 gene homolog. ${ }^{24}$

Conidia formation in fungi is a complicated and tightly regulated process, which is often controlled by different signaling pathways in a given species. Our studies also showed that formation of conidia by A. alternata is regulated by the $\mathrm{G}$ protein-regulated cAMP level.40 Mutational inactivation of a $\mathrm{G} \alpha$ subunit-coding gene $(A a G \alpha l)$ in A. alternata resulted in a severe reduction in conidiation. Exogenous application of cAMP caused decreased conidiation in the wild-type but partially restored conidia formation in the $\mathrm{G} \alpha$-deficient mutant. In striking contrast, conidiation is negatively controlled by cAMP-dependent PKA. ${ }^{41}$ When the cAMP levels are low, the PKA complex, comprising two regulatory and two catalytic subunits, forms a nonfunctional tetramer. The catalytic subunits are separated from the regulatory subunits upon cAMP binds to the regulatory subunits. 42,43 The activated catalytic subunits phosphorylate downstream enzymes or transcriptional regulators. The A. alternata strain impaired for PKA catalytic subunit gene (PKA ${ }^{\text {cat})}$ produces no detectable PKA activity and produces abundant conidia. In contrast, the strain lacking PKA regulatory subunit gene (PKAreg) forms swelling hyphal segments and produces no mature conidia, the phenotypes highly resembling the strain lacking AaFus3. Because impairment of Fus3 did not affect PKA activity and expression of the PKAcat gene and vice versa, it seems that PKA and Fus3 are two different pathways for conidia production. In addition, recent studies revealed that conidiation by $A$. alternata is regulated by the nicotinamide adenine dinucleotide phosphate oxidase complex implicating in the production of reactive oxygen species, the Slt2 MAP kinase, the $\mathrm{Skn} 7$ responsive regulator and the siderophore-mediated iron acquisition. ${ }^{44-46}$ However, the exact interactions between these pathways leading to conidia formation remain largely unknown.

Northern blot analysis revealed that expression of the AaFus3 gene in A. alternata was upregulated by citrus leaf extracts prepared from host (Minneola) or nonhost (rough lemon) leaves. Pathogenicity assayed on detached Minneola leaves inoculated by placing a mycelial mass revealed that the A. alternata strain lacking AaFus3 produced wild-type levels of ACT, yet induced significantly smaller lesions than wild type. The genetically reverted strain produced necrotic lesions comparable to those induced by the wild-type. However, the mutant strain produced wild-type lesions on citrus leaves with wounding prior to inoculation, indicating the requirement of AaFus3 for the penetration process.

Although AaFus3 is not required for resistance to oxidative stress, our studies suggest a possible linkage between the Yap1-regulated

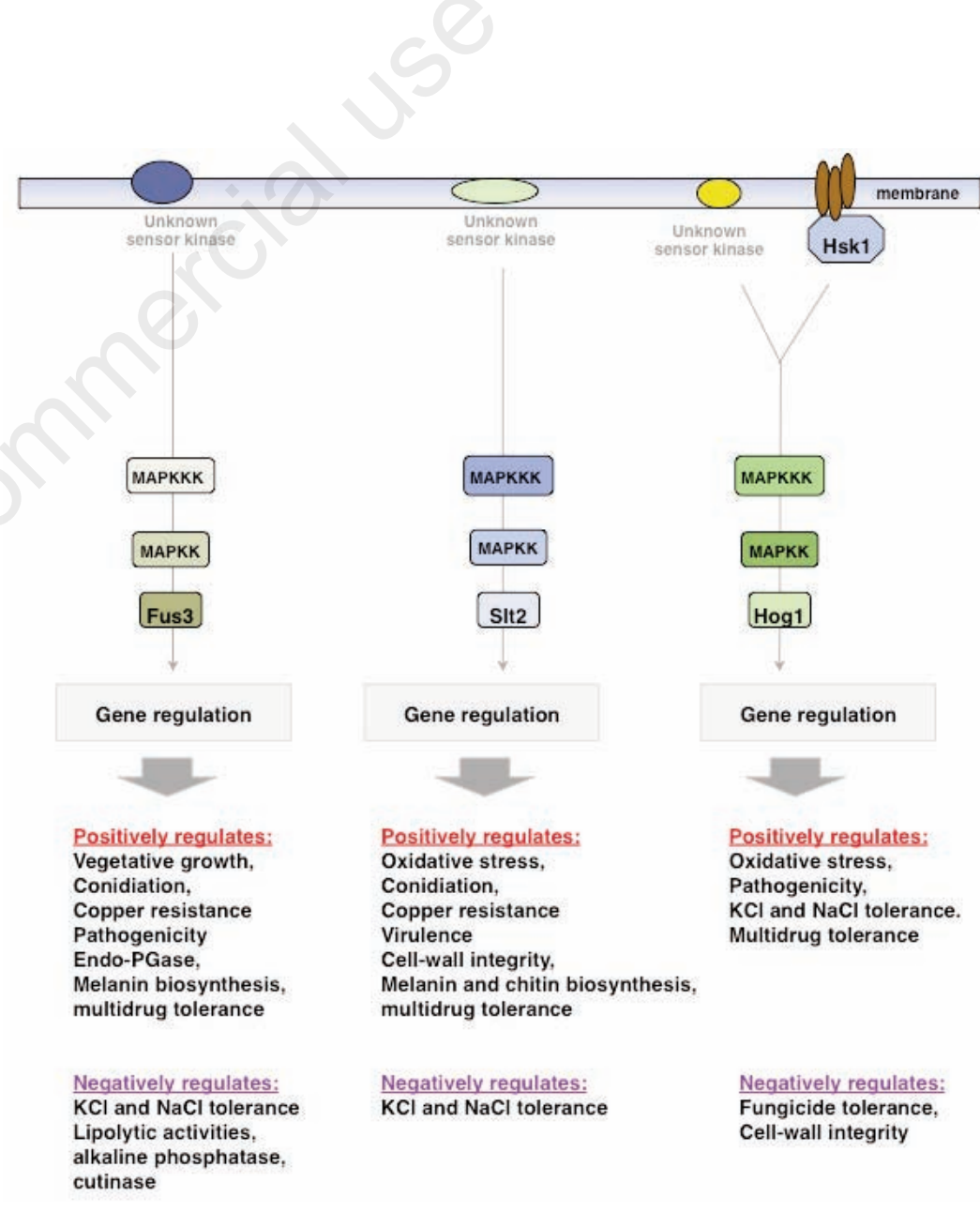

Figure 1. A summary of biological functions of mitogen-activated protein kinase (MAPK)-mediated signaling pathways - the high-osmolarity glycerol (Hog1), the cell wall integrity (Slt2) and the pheromone response (Fus3/Kss1) homologs - in the tangerine pathotype of Alternaria alternata. 
gene expression and a Fus3 MAPK-mediated signaling pathway in A. alternata. Yap1, belonging to the mammalian AP1 family, is a leucine zipper-containing transcriptional activator implicated in cellular responses to stress. $^{47}$ Both AaAP1 (a YAP1 homolog) and AaFus 3 were found to be required for full resistance to 2-chloro-5-hydroxypyridine (CHP), 2,3,5-triiodobenzonic acid (TIBA), diethyl maleate (DEM) and many pyridinecontaining compounds. The AaFus3, but not $A a A P 1$, mutant strain also displayed an increased sensitivity to pyridoxine (vitamin B6) and its derivative pridoxal-5-phosphate. Inactivation of the AaAP1 gene increased phosphorylation of AaFus3; however, application of

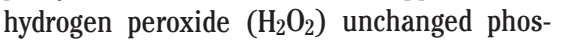
phorylation of AaFus3. ${ }^{39}$ The $\Delta$ yap1 $\Delta$ fus 3 double mutant strain displayed a greater chemical sensitivity compared to the strains mutated at either AaFus3 or AaAP1 alone. Furthermore, TIBA, CHP or DEM promoted accumulation of the $A a A P 1$ and AaFus3 gene transcripts and enhanced AaFus3 phosphorylation. The AaAP1::sGFP (synthetic green fluorescence protein) fusion protein became localized in the nucleus after the treatment of $\mathrm{H}_{2} \mathrm{O}_{2}$, TIBA or CHP. Thus, the AaAP1 and AaFus3 signaling pathways are both required for multidrug resistance, probably by regulating common membrane transporters or enzymes that are directly involved in the efflux or detoxification of toxic substances. Recently, expression of two genes encoding putative major facilitator superfamily (MFS) transporters has been demonstrated to be coordinately up-regulated by AaAP1 and AaFus3.48 Deletion of either of the two MFS transporter-coding genes yielded fungi that displayed an elevated sensitivity to TIBA and CHP (L.-H Chen and H.-C. Tsai, 2012, personal communication). The AaFus3-mediated signaling pathway down-regulates the alkaline phosphatase, cutinase and lipolytic enzymatic activities, ${ }^{49}$ since the impaired strain showed higher levels of those enzymatic activities than the wild-type. However, the $A$. alternata $\Delta$ fus3 mutant accumulated lower endo-PGase activities. AaFus3 has no impact on pectinase, xylanase and cellulase activities. The $\Delta$ yapl mutant strain accumulated wildtype levels of all enzymes tested.

\section{The cell wall integrity-mediat- ed signaling pathway in $A$. alternata}

A. alternata has the yeast Slt2 homolog implicating in maintenance of cell wall integrity. The A. alternata Slt2 (AaSlt2) gene encodes a polypeptide of 416 amino acids, containing a serine/X/threonine (TXY) activation site in the
$\mathrm{N}$ termini, commonly found in the yeast and fungal extracellular signal-regulated kinase (YERK1) subfamily 5 and a protein kinase ATPbinding signature. AaSlt2 is similar to numerous MAP kinases associated with cell wall integrity of fungi. The AaSLT2 MAP kinase protein is most similar to SLT2-like proteins of $A$. brassicicola (AAU11317), Ajellomyce capsulatus (XP_001538584) and C. heterostrophus (ABM54149) showing 85 to $99 \%$ identities. AaSlt2 MAP kinase-mediated signaling pathway has been demonstrated to regulate diverse physiological, developmental and pathological functions in A. alternata (Figure 1). Deletion of AaSlt2 resulted in fungi ( $\Delta$ slt2) showing growth retardation and producing fluffy and white colonies on potato dextrose agar. Unlike the wild-type strain of $A$. alternata, the $\Delta$ sit2 mutant produces globose and less branching hyphae; some of them form hairpin loops at the end of hyphae, suggesting that AaSlt2 plays a critical role in fungal development. 45

The A. alternata Slt2-impaired mutant reduced conidia formation as much as $95 \%$. Conidia formation was restored in strains by transforming $\Delta$ slt2 mutant protoplasts with a functional AaSlt2. Application of $\mathrm{KCl}$ or $\mathrm{NaCl}$ restored vegetative growth but not conidiation by $\Delta$ sit2. In contrast, application of caffeine, sodium dodecyl sulfate, sorbitol, glucose or $\mathrm{H}_{2} \mathrm{O}_{2}$ did not improve vegetative growth and conidia formation of the mutant. Conidia produced by the wild-type are multicellular, obpyriform and contain both vertical and transverse septae. On the other hand, $\Delta$ sit2 mutant produces aberrant, less melanized conidia with fewer vertical septae and thinner cell wall. 45 Compared to wild type, $\Delta$ slt 2 mutant has lower melanin content. The size of conidia produced by wild-type and $\Delta$ sit 2 is also very different. $\Delta$ sit2 mutant produces larger conidia than wild-type. Conidia produced by $\Delta$ slt 2 mutant have less verruculose relative to those of wild type, as examined by scanning electron microscopy. The complementation strain produces conidia similar to those produced by the wild-type.

The $\Delta$ slt2, but not $\Delta$ fus3, mutant were hypersensitive to cell-wall destructing compounds, calcofluor white and Congo red, which interfere with chitin polymerization. $\Delta$ sit2 mutant has lower cell wall chitin content compared to the levels measured in the wild type and the genetically reverted strains. $\Delta$ sit 2 mutant released protoplasts at a rate and magnitude significantly greater than the wild-type and the complementation strains in the presence of an enzymatic mixture containing driselase, lyticase, $\beta$-D-glucanase and $\beta$-glucuronidase, ${ }^{45}$ confirming further that AaSit2 plays an important role in maintaining cell wall integrity. Unlike $A$. alternata, the $B$. cinerea and the $\mathrm{Col}$. lagenarium slt 2 mutants were unchanged in sensitivity to cell wall-degrading enzymes or Calcofluor white compared to their parental strains. 50,51

The Slt2 MAPK signaling pathways are well conserved in eukaryotes. However, they may have different functions within and between species. For examples, Slt2 homologs are required for maintaining the integrity of cell walls in A. brassicicola, Cl. purpurea, $M$. grisea, Fusarium graminearum or Aspergillus nidu-

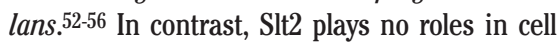
wall integrity in $B$. cinerea, Col. lagenarium or My. Graminicola. ${ }^{50,51,57}$ The A. brassicicola mutant lacking a Slt2 MAP kinase is more sensitive to $\mathrm{H}_{2} \mathrm{O}_{2}$ than its progenitor; 55,58 the $B$. cinerea slt2 mutant is hypersensitive to fludioxonil and paraquat.51 The A. alternata $\Delta$ slt2 mutant displayed wild-type sensitivity to $\mathrm{H}_{2} \mathrm{O}_{2}$ and dicarboximide (iprodione and vinclozolin) and phenylpyrrole (fludioxonil) fungicides. Similar to fungal strain lacking AaFus3 or $A a A P 1, \triangle$ slt2 mutant displayed an increased sensitivity to CHP, TIBA or compounds containing pyridine or benzene backbones. ${ }^{45}$ Fungal strain lacking a G $\alpha$ subunit- or a PKA catalytic subunitcoding gene displayed wild-type sensitivity to those compounds. In fungi, the functions of membrane-bound transporters or pumps, such as the ATP-binding cassette transporters and the MFS transporters are often contributable to multidrug resistance. 59 We speculate that those signaling pathways may directly or indirectly regulate some of the transporters and facilitate efflux of the toxic materials in A. alternata.

Slt2 homologs have been shown to be required for the production of melanin by Neurospora crassa and $C$. heterostrophus and for the production of deoxynivalenol by $F$. graminearum. $12,53,60$ We also found that the $A$. alternata Slt2 is involved in regulating biosynthesis of secondary metabolites, because $\Delta$ slt2 mutant accumulated lower levels of ACT toxin and melanin. ${ }^{45}$ The levels of ACT toxin and melanin were restored in a strain that expresses a wild-type copy of AaSlt2.

AaSlt2 is required for full virulence of $A$. alternata on citrus. Fungal virulence performed on detached Minneola leaves sprayed uniformly with conidial suspension revealed that $\Delta$ slt2 mutant produced significantly fewer and smaller necrotic lesions than the wildtype. This pathogenic impairment could be in part due to the reduced production of ACT toxin by $\Delta$ sit 2 mutant. The reduction of fungal virulence resulting from the deletion of AaSlt2 could also be due to slow growth and abnormality of hyphal extension of the mutant. Slt2 homologs also play an important function in pathogenicity/virulence in a number of phytopathogenic fungi. These include $B$. cinerea, Col. lagenarium, Cl. purpurea, F. graminearum, M. grisea, C. heterostrophus, My. graminicola and $A$. brassicicola, ${ }^{50-58}$ as well as the human pathogens, Can. albicans and Cryptococcus neoformans. 61,62 


\section{The high osmolarity glycerol mitogen-activated protein kinase-mediated signaling pathway in A. alternata}

In S. cerevisiae, the activated Hogl pathway facilitates glycerol accumulation, allowing the yeast to cope with high osmolarity induced by the surrounding environment. ${ }^{63-66}$ The function of Hogl is regulated by phosphorylation via a two-component histidine kinase (HSK)mediated signaling pathway. ${ }^{64,66,67} \mathrm{~S}$. cerevisiae has a single HSK, Sln1p, which is required for osmotic adaption via an Ypdlp containing a histidine (His) phosphotransfer (HPt) domain and two response regulators (RR), Ssklp and Skn7p.68-70 All fungal HSKs identified have both a histidine kinase and a RR domains. ${ }^{63,65,71}$ Under normal osmolarity, the Sln1p kinase is spontaneously phosphorylated at a conserved histidine residue. The phosphate is passed down to Ypdlp and then Ssklp or Skn7p in a pattern of His-Asp-His-Asp. 67 The phosphorylated Ssklp is inactive and fails to activate the Hog1 MAP kinase pathway. When Ssk1p is dephosphorylated, it is able to activate the Hog1 pathway that, in turn, promotes glycerol accumulation, allowing the yeast to counteract high osmolarity. In contrast, the phosphorylated Skn7p is active and capable of regulating the genes whose products are responsible for low osmolarity. 68,70 In addition, $S$. cerevisiae uses a Sholp protein, independent of HSK, to counteract osmotic stress.72,73 HSKmediated signals have long been thought to be transduced primarily via the Hog1 MAP kinase pathway. ${ }^{74}$ With the exception of $S$. cerevisiae, all fungi have multiple HSK signaling genes, ranging from three HSK genes in the fission yeast Schizosaccharomyces pombe to as many as 21 HSK genes in the filamentous fungus $C$. heterostrophus. 75 This signaling pathway has been implicated in osmotic and oxidative responses, hyphal development, toxin biosynthesis, virulence, as well as sensitivity to dicarboximide and phenylpyrrole fungicides in diverse fungal species, including $N$. crassa, Bot. fuckeliana, A. alternata, A. brassicicola, Cl. purpurea, M. grisea, My. Graminicola, C. heterostrophus and $F$. graminearum. ${ }^{76-89}$

The A. alternata Hog1 (AaHog1), analogous to the yeast Hog1, has a distinct phosphorylation motif ( $\underline{\mathrm{TG}} \underline{\mathrm{Y}})$ required for the hyperosmolarity response. ${ }^{5}$ AaHogl also has a protein kinase ATP-binding region, a MAP kinase and a serine/threonine protein kinase active site. The A. alternata Hskl (AaHskl), analogous to fungal group III two-component histidine kinase, has a HAMP (histidine kinase, addenylate cyclase, methyl binding protein, and phosphatase) domain and a dimerization/phosphoacceptor domain. Unlike the yeast histidine kinase Sln1p, AaHsk1 has no transmembrane domains.

AaHOG1 plays a critical role in cellular resistance to oxidative and salt stresses in $A$. alternata (Figure 1). Inactivation of the Hog1 homolog by targeted gene disruption in $A$. alternata produced fungi that showed an increased sensitivity to $\mathrm{KCl}$ and $\mathrm{NaCl}$ salts, menadione, tert-butyl-hydroxyperoxide, $\mathrm{H}_{2} \mathrm{O}_{2}$, TIBA and CHP. ${ }^{90}$ Hog 1 mutant displayed wildtype sensitivity to glucose, sucrose, sorbitol or mannitol. In contrast, inactivation of the $A$. alternata $H s k 1$ gene produced fungi that showed an increased sensitivity to sucrose, mannitol, glucose, sorbitol, TIBA or CHP. $\Delta$ hskl mutant displayed wild-type sensitivity to tert-butyl-hydroxyperoxide, $\mathrm{H}_{2} \mathrm{O}_{2}, \mathrm{KCl}$ or $\mathrm{NaCl}$. Hence, AaHskl plays a regulatory role in osmotic adaption, specifically to sugar osmoticants, without the involvement of the AaHog1mediated pathway. AaHog1, independent of AaHsk1, confers cellular resistance to salts and oxidative stress. Although $\Delta$ hogl mutant displayed wild-type sensitivity to sugars, these osmoticants promoted AaHogl phosphorylation and subsequently nuclear localization in the $\Delta$ hskl mutant background. ${ }^{90}$ In the wildtype background, sugar osmoticants did not impact AaHog1 phosphorylation and did not facilitate nuclear localization of AaHogl. Pathogenicity assays revealed that AaHog1 is required for fungal pathogenicity, but $A a H s k 1$ is dispensable for pathogenicity. The AaHog1impaired mutants are non-pathogenic, producing no necrotic lesions on Minneola leaves. Inactivation of the AaHogl or AaHskl gene did not influence the production of host-selective ACT toxin by $A$. alternata. It appears that $A$. alternata recruits AaHskl and AaHogl to perform a unique function in resistance to sugar osmoticants and salt stress, respectively. $S$. cerevisiae employs the two-component histidine kinase Slnlp and the membrane protein Sholp, both via regulation of the Hog1 pathway, to counteract osmotic stress. ${ }^{72}$ In contrast, the filamentous fungus $A$. nidulans Sholp homolog is not required for cellular response to osmotic stress..$^{91}$ Recently, a Sholp homolog was cloned and inactivated in the tangerine pathotype of $A$. alternata, revealing no role in osmotic adaption (L.H. Chen, 2012, unpublished). Disruption of the Ssk1p homolog (a responsive regulator upstream of Hog1) in $A$. alternata resulted in fungi that displayed an elevated sensitivity to $\mathrm{H}_{2} \mathrm{O}_{2}$, tert-butyl hydroperoxide, menadione, salts, but not glucose, phenotypes resembling those seen with the $\Delta$ hog1 mutant (L.H. Chen, 2012, unpublished).

Unlike Slt2, AaHog1 appears to have a negatively regulatory role in the maintenance of cell wall integrity. A fungal strain lacking AaHog1, but not AaHskl, was highly resistant to cellwall degrading enzymes, such as driselase, $\beta$ -
D-glucanase, $\beta$-glucuronidase and lyticase, producing no protoplasts. Moreover, AaHogl and AaFus3 have an opposite role in terms of $\mathrm{KCl}$ or $\mathrm{NaCl}$ tolerance (Figure 2). As stated above, $\Delta$ fus 3 mutant displayed an increased resistance to $\mathrm{KCl}$ and $\mathrm{NaCl}$, whereas $\Delta \mathrm{hog} 1$ mutant displayed an increased sensitivity to them. In $S$. cerevisiae, Hogl has also been shown to negatively regulate the Fus3/Kss1 signaling cascade during hyperosmotic stress. 13,14

AaHog1 and AaHsk1 have shared functions as well, because fungi impaired for AaHogl or AaHskl were more resistant to dicarboximide and phenylpyrrole fungicides than the wildtype. Compared to the resistance seen with the $\Delta$ hsk1 mutants, the $\Delta$ hogl mutant was barely resistant to these fungicides, implicating that AaHsk1 is the key regulator for sensitivity to dicarboximide and phenylpyrrole fungicides..$^{90}$ Nuclear localization is important for proper functions of Hog1.92 Under normal conditions, the AaHogl protein was phosphorylated at low levels in the wild-type strain of $A$. alternata. Exposure to iprodione or fludioxonil fungicide, TIBA, CHP, $\mathrm{NaCl}$ or $\mathrm{H}_{2} \mathrm{O}_{2}$ enhanced AaHog1 phosphorylation and nuclear localization.

\section{The two component histidine kinase-Skn7 signaling pathway in A. alternata}

All living cells have different signaling transduction pathways to perceive changes in their environments and to adjust physiological and developmental processes. ${ }^{93-99} S$. cerevisiae has two major activation mechanisms - the Sln1p-Ypdlp-Ssklp-Hog1 and the Sholp-mediated pathways - in response to osmotic and oxidative stress. Similar to Ssk1 response regulator, Skn7 is a transcription downstream regulator of Sln1p (Figure 2). Skn7p is phosphorylated, specifically occurring at the Asp (D427), under conditions of low osmolarity. ${ }^{70,100}$ In contrast, under low turgor conditions, Sln1p kinase is phosphorylated and subsequently activates Ssklp by a phosphorelay mechanism. The phosphorylated Ssklp is inactive and unable to activate the Hog1 MAP kinase pathway.

In response to oxidative stress, Skn7p is not modulated by the SIn1p-mediated phosphorylation. ${ }^{68,101}$ Under oxidative stress, Skn7p is phosphorylated at serine or threonine residue and forms a heterodimer with the stress responsive transcription regulator Yap1.100,102,103 Interaction between Skn7 and Yap1 regulates numerous genes associated with oxidative stress response.101,104-106 However, Yap1 confers resistance to cadmium resistance, apparently bypassing Skn7p.107 
Skn7p could interact with the calcium responsive activator, the heat-shock transcription factor, the Rhol GTPase or the cell cycle transcription regulator under different physiological conditions. ${ }^{108-111}$ In fungi, Skn7 has been demonstrated to be required for sporulation, fungicide sensitivity, cell wall biosynthesis, oxidative stress adaptation, osmotic stress response, cell cycle, sexual mating and pathogenicity/virulence. ${ }^{101,109,112-120}$ The $A$. alternata Skn7 homolog was cloned and functionally inactivated, revealing a close association of Skn7 response regulator and resistance to osmotic and oxidative stress, conidiation, conidial morphology and fungicide sensitivity. 44 The Ssk1-Hogl pathway, independent of Hsk1 (a Sln1p ortholog), confers resistance primarily to salts and oxidative stress, whereas the Hsk1-Skn7 pathway is responsible for sugar-induced osmotic and oxidative stress (Figure 2).

Genetic analyses reveal that disruption of the $A$. alternata $S k n 7$ gene produced fungi that were more sensitive to $\mathrm{H}_{2} \mathrm{O}_{2}$, tert-butyl hydroperoxide and cumyl peroxide, but not to the superoxide-generating compounds (menadione, potassium superoxide and diamide). $\Delta$ skn7 mutant also displayed an increased sensitivity to glucose, mannitol, sucrose and sorbitol. However, the $\Delta s k n 7$ mutant displayed wild-type sensitivity to $\mathrm{NaCl}$ and $\mathrm{KCl}$ salts. The $\Delta$ skn7 mutant displayed an elevated resistance to dicarboximide (iprodione and vinclozolin) and phenylpyrrole fungicides at levels between the AaHsk1 and the AaHogl mutant strains. A fungal strain impaired for $S s k 1$, a responsive regulator upstream of $\mathrm{Hog} 1$, displayed an increased sensitivity to these fungicides at levels similar to those seen with the $\Delta$ skn7 mutant (L.H. Chen, 2012, unpublished), indicating that both Ssk1 and Skn7 are involved in fungicide sensitivity. Fungal strain carrying skn7/hog1 double mutations exhibited fungicide resistance, similar to the strain with a single AaHskl gene mutation. ${ }^{44}$ The results indicate that the signals associated with fungicide sensitivity are passed from AaHskl down to both Skn7- and Ssk1-HOG-mediated pathways. Pathologically, the A. alternata Skn7 and Sskl-Hoglsinglaing pathways are both

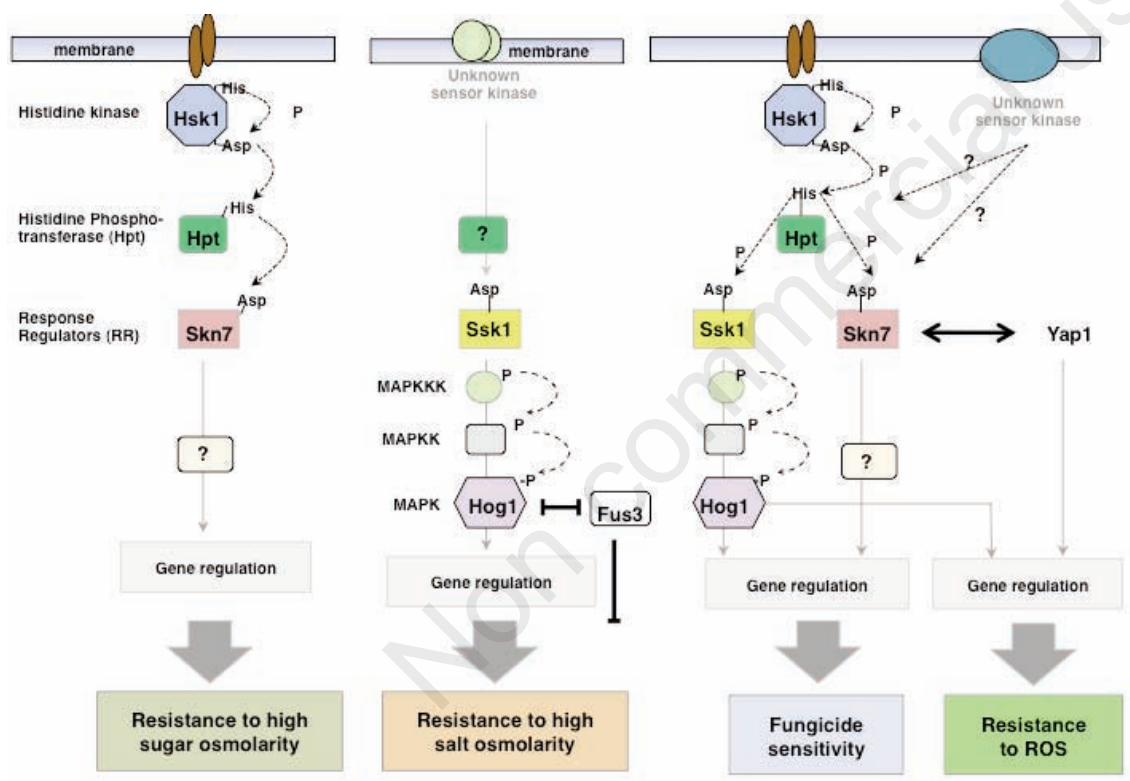

Figure 2. Proposed signaling pathways involving a two-component histidine kinase (Hsk1), a histidine phosphotransferase (Hpt) and two responsive regulators (RR), Skn7 and Ssk1, for resistance to high osmolarity and fungicide sensitivity in the necrotrophic fungal pathogen Alternaria alternata. Upon sensing high levels of sugars, Hsk1 is phosphorylated and the phosphate is transferred to downstream Hpt and then $\operatorname{Skn} 7 \mathrm{p}$ in a pattern of histidine (His)-aspartate (Asp)-His-Asp. Skn7 in turn regulates downstream signaling pathways and eventually produces a change in gene expression. Hsk1 plays no role in resistance to osmotic stress induced by high levels of salts. Unknown kinases, instead of Hsk1, could phosphorylate the Ssk1 regulator via a phosphorelay mechanism and activate the high osmolarity-glycerol 1 (Hog1) MAP kinase pathway. In contrast to Hog1, the Fus3 MAP kinase plays a negative regulatory role for salt tolerance. Hog1 and Hsk1 have shared functions as well, because the $A$. alternata mutant strains lacking Hsk1, Ssk1, Skn7 or Hog1 are more sensitive to dicarboximide and phenylpyrrole fungicides than wild-type. Hsk1 is not required for resistance to reactive oxygen species (ROS). Upon sensing oxidative stress, both Ssk1-Hog1 and Skn7, perhaps activated by unknown kinases, independently regulate the genes required for detoxifying toxic ROS. Skn7 may interact with the redox responsive regulator Yap1 for ROS resistance. required for fungal colonization and lesion development in susceptible cultivars of citrus. ${ }^{44,90}$ Mutation of the $A$. alternata Hsk1 gene did not impact fungal pathogenicity, confirming further that $A$. alternata employs specialized or shared regulatory interactions among different signaling pathways for diverse physiological and pathological functions.

\section{Conclusions}

MAPK-mediated signaling cascades play subtle regulatory roles during vegetative growth and conidia formation, for the production of hydrolytic enzymes and melanin, and for resistance to fungicides, osmotic stress and a broad spectrum of structurally diverse compounds in A. alternata. Those signaling pathways also have a substantial contribution to fungal pathogenicity and to effective penetration and tissue colonization of citrus hosts. Different MAPK pathways may interact in a cooperative or antagonistic manner, thus diversifying their specificities. The biological roles for MAPK signaling pathways in A. alternata are established, but many questions remain to be answered. How $A$. alternata perceives and responds to environmental stimuli will be the key areas for future investigation. Identifying membrane-bound sensor kinases upstream each of the pathways and downstream target proteins will help understand the complex nature of MAPK signaling pathways in relation to various biological and pathological processes in this important fungal pathogen of citrus.

\section{References}

1. Gustin MC, Albertyn J, Alexander M, Davenport K. MAP kinase pathways in the yeast Saccharomyces cerevisiae. Microbiol Mol Biol Rev 1998;62:1264-300.

2. Qi M, Elion EA. Map kinase pathways. J Cell Sci 2005;118:3569-72.

3. Pelech SJ, Sanghera JS. MAP kinases: charting the regulatory pathways. Science 1992;257:1355-6.

4. Robinson MJ, Cobb MH. Mitogen-activated protein kinase pathways. Curr Opin Cell Biol 1997;9:180-6.

5. Kültz D. Phylogenetic and functional classification of mitogen- and stress-activated protein kinases. J Mol Evol 1998;46: 571-88.

6. Schwartz MA, Madhani HD. Principles of MAP kinase signaling specificity in Saccharomyces cerevisiae. Annu Rev Genet 2004;38:725-48. 
7. Xu JR. MAP kinases in fungal pathogens. Fungal Genet Biol 2000;31:137-52.

8. Zhou X, Mehrabi R, Xu JR. Mitogen-activated protein kinase pathways and fungal pathogenesis. Eukaryot Cell 2007;6:170114.

9. Bardwell L. Mechanisms of MAPK signaling specificity. Biochem Soc Transact 2006;34:837-41.

10. Bahn YS, Xue C, Idnurm A, et al. Sensing the environment: lessons from fungi. Nat Rev Microbiol 2007;5:57-69.

11. Kronstad J, De Maria A, Funnell D, et al. Signalling via cAMP in fungi: interconnections with mitogen-activated protein kinase pathways. Arch Microbiol 1998;170:395-404.

12. Eliahu N, Igbaria A, Rose MS, et al. Melanin biosynthesis in the maize pathogen Cochliobolus heterostrophus depends on two mitogen-activated protein kinases, CHk1 and Mps1, and the transcription factor Cmr1. Eukaryot Cell 2007;6:421-9.

13. O'Rourke SM, Herskowitz I. The Hog1 MAPK prevents cross talk between the HOG and pheromone response MAPK pathways in Saccharomyces cerevisiae. Genes Dev 1998;12:2874-86.

14. Shock TR, Thompson J, Yates JR III, Madhani HD. Hog1 mitogen-activated protein kinase (MAPK) interrupts signal transduction between the Kss1 MAPK and the Tecl transcription factor to maintain pathway specificity. Eukaryot Cell 2009;8:606-16.

15. Hatta R, Ito K, Hosaki Y, et al. 2002. The conditionally dispensable chromosome controls host-specific pathogenicity in the fungal plant pathogen Alternaria alternata. Genetics 2002;161:59-70.

16. Ito K, Tanaka T, Hatta R, et al. Dissection of the host range of the fungal plant pathogen Alternaria alternata by modification of secondary metabolism. Mol Microbiol 2004;52:399-411.

17. Kohmoto K, Akimitsu K, Otani $\mathrm{H}$. Correlation of resistance and susceptibility of citrus to Alternaria alternata with sensitivity to host-specific toxins. Phytopathology 1991;81:719-22.

18. Nishimura S, Kohmoto K. Host-specific toxins and chemical structures from Alternaria species. Annu Rev Phytopathol 1983;21:87-116.

19. Kohmoto K, Itoh Y, Shimomura N, et al. Isolation and biological activities of two host-specific toxins from tangerine pathotype of Alternaria alternata. Phytopathology 1993;83:495-502.

20. Otani H, Kohmoto K, Kodama M. Alternaria toxins and their effects on host plants. Can J Bot 1996;73:S453-8.

21. Madhani HD, Fink GR. The control of fila- mentous differentiation and virulence in fungi. Trends Cell Biol 1998;8:348-53.

22. Xu J-R, Hamer JE. MAP kinase and cAMP signaling regulate infection structure formation and pathogenic growth in the rice blast fungus Magnaporthe grisea. Genes Dev. 1996;10:2696-706.

23. Choi ES, Chung HJ, Kim MJ, et al. Characterization of the ERK homologue CpMK2 from the chestnut blight fungus Cryphonectria parasitica. Microbiology 2005;151:1349-58.

24. Cho Y, Cramer RA Jr, Kim KH, et al. The Fus3/Kss1 MAP kinase homolog Amk1 regulates the expression of genes encoding hydrolytic enzymes in Alternaria brassicicola. Fungal Genet Biol 2007;44:54353.

25. Cousin A, Mehrabi R, Guilleroux M, et al. The MAP kinase-encoding gene MgFUS3 of the non-appressorium phytopathogen Mycosphaerella graminicola is required for penetration and in vitro pycnidia formation. Mol Plant Pathol 2006;7:269-78.

26. Di Pietro A, García-Macelra FI, Méglecz E, Roncero IG. A MAP kinase of the vascular wilt fungus Fusarium oxysporum is essential for root penetration and pathogenesis. Mol Microbiol 2001;39:1140-52.

27. Igbaria A, Lev S, Rose MS, et al. Distinct and combined roles of the MAP kinases of Cochliobolus heterostrophus in virulence and stress responses. Mol Plant Microbe Interact 2008;21:769-80.

28. Jenczmionka NJ, Maier F, Lösch AP, Schäfer W. Mating, conidiation and pathogenicity of Fusarium graminearum, the main causal agent of the head-blight disease of wheat, are regulated by the MAP kinase gpmk1. Curr Genet 2003;43:87-95.

29. Jenczmionka NJ, Schäfer W. The Gpmk1 MAP kinase of Fusarium graminearum regulates the induction of specific secreted enzymes. Curr Genet 2005;47:29-36.

30. Lev S, Sharon A, Hadar R, et al. A mitogen-activated protein kinase of corn leaf pathogen Cochliobolus heterostrophus is involved in conidiation, appressorium formation, and pathogenicity: diverse roles for mitogen-activated protein kinase homologs in foliar pathogens. Proc Natl Acad Sci U S A 1999;96:13542-7.

31. Moriwaki A, Kihara J, Mori C, Arase S. A MAP kinase gene, BMK1, is required for conidiation and pathogenicity in the rice leaf spot pathogen Bipolaris oryzae. Microbiol Res 2007;162:108-14.

32. Rauyaree $\mathrm{P}$, Ospina-Giraldo MD, Kang S, et al. Mutations in VMK1, a mitogen-activated protein kinase gene, affect microsclerotia formation and pathogenicity in Verticillium dahlia. Curr Genet 2005;48:109-16.

33. Ruiz-Roldán MC, Maier FJ, Schäfer W.
PTK1, a mitogen-activated protein kinase gene, is regulated for conidiation, appressorium formation, and pathogenicity of Pyrenophora teres on Barley. Mol Plant Microbe Interact 2001;14:116-25.

34. Solomon PS, Waters ODC, Simmonds J, et al. The Mak2 MAP kinase signal transduction pathway is required for pathogenicity in Stagonospora nodorum. Curr Genet 2005;48:60-8.

35. Takano Y, Kikichi T, Kubo Y, et al. The Colletotrichum lagenarium MAP kinase gene CMK1 regulates diverse aspects of fungal pathogenesis. Mol Plant Microbe Interact 2000;13:374-83.

36. Urban M, Mott E, Farley T, HammondKosack K. The Fusarium graminearum MAP1 gene is essential for pathogenicity and development of perithecia. Mol Plant Pathol 2003;4:347-59.

37. Zheng L, Cambell M, Murphy J, et al. 2000. The BMP1 gene is essential for pathogenicity in the gray mold fungus Botrytis cinerea. Mol Plant Microbe Interact 2000;13:724-32.

38. Guhad FA, Jensen HE, Aalback B, et al. Mitogen-activated protein kinase-defective Candida albicans is avirulent in a novel model of localized murine candidiasis. FEMS Microbiol Lett 1998;166:135-9.

39. Lin CH, Yang SL, Wang NY, Chung KR. The FUS3 MAPK signaling pathway of the citrus pathogen Alternaria alternata functions independently or cooperatively with the fungal redox-responsive AP1 regulator for diverse developmental, physiological and pathogenic processes. Fungal Genet Biol 2010;47:381-91.

40. Wang NY, Lin CH, Chung KR. A G $\alpha$ subunit gene is essential for conidiation and potassium efflux but dispensable for pathogenicity of Alternaria alternata in citrus. Curr Genet 2010;56:43-51.

41. Tsai HC, Yang SL, Chung KR. Cyclic AMPdependent protein kinase A negatively regulates conidia formation by the tangerine pathotype of Alternaria alternata. World J Microbiol Biotechnol 2012;29:289. 300 .

42. D'Souza CA, Heitman J. Conserved cAMP signaling cascades regulate fungal development and virulence. FEMS Microbiol Rev 2001;25:349-64.

43. Gerits N, Kostenko S, Shiryaev A, et al. Relations between the mitogen-activated protein kinase and the cAMP-dependent protein kinase pathways: comradeship and hostility Cell Signal 2008;20:1592607.

44. Chen LH, Lin CH, Chung KR. Roles for SKN7 response regulator in stress resistance, conidiation and virulence in the citrus pathogen Alternaria alternata. Fungal Genet Biol 2012;49:802-13. 
45. Yago JI, Lin $\mathrm{CH}$, Chung KR. The SLT2 mitogen-activated protein kinase-mediated signaling pathway governs conidiation, morphogenesis, fungal virulence and production of toxin and melanin in the tangerine pathotype of Alternaria alternata. Mol Plant Pathol 2011;12:65365 .

46. Yang SL, Chung KR. The NADPH oxidasemediated production of $\mathrm{H} 2 \mathrm{O} 2$ and resistance to oxidative stress in the necrotrophic pathogen Alternaria alternata of citrus. Mol Plant Pathol 2012;13:900 14.

47. Moye-Rowley WS. Regulation of the transcriptional response to oxidative stress in fungi: similarities and differences. Eukaryot Cell 2003;2:381-9.

48. Lin CH, Yang SL, Chung KR. Cellular responses required for oxidative stress tolerance, colonization and lesion formation by the necrotrophic fungus Alternaria alternata in citrus. Curr Microbiol 2011;62:807-15.

49. Lin CH, Yang SL, Chung KR. The YAP1 homolog-mediated oxidative stress tolerance is crucial for pathogenicity of the necrotrophic fungus Alternaria alternata in citrus. Mol Plant Microbe Interact 2009;22:942-52.

50. Kojima K, Kikuchi T, Takano Y, et al. The mitogen-activated protein kinase gene MAF1 is essential for the early differentiation phase of appressorium formation in Colletotrichum lagenarium. Mol PlantMicrobe Interact 2002;15:1268-76.

51. Rui 0, Hahn M. The Slt2-type MAP kinase Bmp3 of Botrytis cinerea is required for normal saprotrophic growth, conidiation, plant surface sensing and host tissue colonization. Mol Plant Pathol 2007;8:173-84.

52. Fujioka T, Mizutani 0, Furukawa $\mathrm{K}$, et al. MpkA-dependent and -independent cell wall integrity signaling in Aspergillus nidulans. Eukaryot Cell 2007;6:1497-510.

53. Hou Z, Xue C, Peng Y, et al. A mitogenactivated protein kinase gene (MGV1) in Fusarium graminearum is required for female fertility, heterokaryon formation, and plant infection. Mol Plan Microbe Interact 2002;15:1119-27.

54. Mey G, Held K, Scheffer J, et al. 2002. CPMK2, an SLT2-homologous mitogenactivated protein (MAP) kinase, is essential for pathogenesis of Claviceps purpurea on rye: evidence for a second conserved pathogenesis-related MAP kinase cascade in phytopathogenic fungi. Mol Microbiol 2002;46:305-18.

55. Scott DC. The cell wall integrity-associated MAP kinase homolog, AbSlt2 in the necrotrophic fungus Alternaria brassicicola is required for pathogenicity of Brassicas; Master Thesis. Virginia
Polytechnic Institute and State University; 2009. Available from: http:/scholar.lib.vt.edu/theses/available/e td-02102009-234303/

56. Xu JR, Staiger CJ, Hamer JE. Inactivation of the mitogen-activated protein kinase MPS1 in the rice blast fungus prevents penetration of host cells but allows activation of plant defense responses. Proc Natl Acad Sci U S A 1998;95:12713-18.

57. Mehrabi $\mathrm{R}$, van der Lee T, Waalwijk C, Kema GHJ. MgSlt2, a cellular integrity MAP kinase gene of the fungal wheat pathogen Mycosphaerella graminicola, is dispensable for penetration but essential for invasive growth. Mol Plant Microbe Interact 2006;19:389-98.

58. Joubert A, Bataille-Simoneau N, Campion $\mathrm{C}$, et al. Cell wall integrity and high osmolarity glycerol pathways are required for adaptation of Alternaria brassicicola to cell wall stress caused by brassicaceous indolic phytoalexins. Cell Microbiol 2010;13:62-80.

59. Gulshan K, Moye-Rowley WS. Multidrug resistance in fungi. Eukaryot Cell 2007;6:1933-42.

60. Park G, Pan S, Borkovich KA. Mitogenactivated protein kinase cascade required for regulation of development and secondary metabolism in Neurospora crassa. Eukaryot Cell 2008;7:2113-22.

61. Kraus PR, Fox DS, Cox GM, Heitman J. The Cryptococcus neoformans MAP kinase Mpkl regulates cell integrity in response to antifungal drugs and loss of calcineurin function. Mol Microbiol 2003;48:1377-87.

62. Monge RA, Román E, Nombela C, Pla J. The MAP kinase signal transduction network in Candida albicans. Microbiology 2006;152:905-12.

63. Santos JL, Shiozaki K. Fungal histidine kinases. Sci STKE 2001;98:re1.

64. Wurgler-Murphy SM, Saito H. Two-component signal transducers and MAPK cascades. Trends Biochem Sci 1997;22:172-6.

65. West AH, Stock AM. Histidine kinases and response regulator proteins in two-component signaling systems. Trends Biochem Sci 2001;26:369-76.

66. Kruppa M, Calderone R. Two-component signal transduction in human fungal pathogens. FEMS Yeast Rev 2006;6:14959.

67. Thomason P, Kay R. Eukaryotic signal transduction via histidine-aspartate phosphorelay. J Cell Sci 2000;113:3141-50.

68. Li S, Ault A, Malone CL, et al. The yeast histidine protein kinase, Sln1p, mediates phosphotransfer to two response regulators, Ssklp and Skn7p. EMB0 J 1998; 17:6952-62.

69. Hoch JA. Two-component and phosphore- lay signal transduction. Curr Opin Microbiol 2000;3:165-70.

70. Posas F, Wurgler-Murphy SM, Maeda T, et al. Yeast HOG1 MAP kinase cascade is regulated by a multistep phosphorelay mechanism in the SLN1-YPD1-SSK1 "two-component" osmosensor. Cell 1996; 86:865-75.

71. Wolanin PM, Thomason PA, Stock JB. Histidine protein kinases: key signal transducers outside the animal kingdom. Genome Biol 2002;3:3013.1-8.

72. Maeda T, Wurgler-Murphy SM, Saito H. A two-component system that regulates an osmosensing MAP kinase cascade in yeast. Nature 1994;369:242-5.

73. Westfall PJ, Ballon DR, Thorner J. When the stress of your environment makes you go HOG wild. Science 2004;306:1511-2.

74. Tao W, Deschenes RJ, Fassler JS. Intracellular glycerol levels modulate the activity of SIn1p, a Saccharomyces cerevisiae two-component regulator. J Biol Chem 1999;274:360-7.

75. Catlett NL, Yoder OC, Turgeon BG. Wholegenome analysis of two-component signal transduction genes in fungal pathogens. Eukaryot Cell 2003;6:1151-61.

76. Alex LA, Borkovich KA, Simon MI. Hyphal development in Neurospora crassa: Involvement of a two-component histidine kinase. Proc Natl Acad Sci U S A 1996;93:3416-21.

77. Avenot H, Simoneau P, Iacomi-Vasilescu B, Bataillé-Simoneau N. Characterization of mutations in two-component histidine kinase gene AbNIK1 from Alternaria brassicicola that confer high dicarboximide and phenylpyrrole resistance. Curr Genet 2005;47:234-43.

78. Cui W, Beever RE, Parkes SL, et al. As osmosensing histidine kinase mediates dicarboximide fungicide resistance in Botryotinia fuckeliana (Botrytis cinerea). Fungal Genet Biol 2002;36:187-98.

79. Dongo A, Bataillé-Simoneau N, Campion $\mathrm{C}$, et al. The group III two-component histidine kinase of filamentous fungi is involved in the fungicidal activity of the bacterial polyketide ambruticin. Appl Environ Microbiol 2009;75:127-34.

80. Dry IB, Yuan KH, Hutton DG. Dicarboximide resistance in field isolates of Alternaria alternata is mediated by a mutation in a two-component histidine kinase gene. Fungal Genet Biol 2004;41:102-8.

81. Kojima K, Takano Y, Yoshimi A, et al. Fungicide activity through activation of a fungal signaling pathway. Mol Microbiol 2004;53:1785-96.

82. Motoyama T, Kadokura K, Ohira T, et al. A two-component histidine kinase of the rice blast fungus is involved in osmotic 
stress response and fungicide action. Fungal Genet Biol 2005;42:200-12.

83. Mehrabi R, Zwiers LH, de Waard MA, Kema GHJ. MgHogl regulates dimorphism and pathogenicity in the fungal wheat pathogen Mycosphaerella graminicola. Mol Plant-Microbe Interact 2006;11:1262-9.

84. Nathues E, Jörgens C, Lorenz N, Tudzynski P. The histidine kinase CpHK2 has impact on spore germination, oxidative stress and fungicide resistance, and virulence of the ergot fungus Claviceps purpurea. Mol Plant Pathol 2007;8:653-65.

85. Ochiai N, Tokai T, Nishiuchi T, et al. Involvement of the osmosensor histidine kinase and osmotic stress-activated protein kinases in the regulation of secondary metabolism in Fusarium graminearum. Biochem Biophys Res Commun 2007:363:639-44.

86. Oide S, Liu J, Yun SH, et al. Histidine kinase two-component response regulator proteins regulate reproductive development, virulence, and stress responses of the fungal cereal pathogens Cochliobolus heterostrophus and Gibberella zeae. Eukaryot Cell 2010;9: 1867-80.

87. Rispail N, Di Pietro A. The two-component histidine kinase Fhk1 controls stress adaptation and virulence of Fusarium oxysporum. Mol Plant Pathol 2010;11:395407.

88. Viaud M, Fillinger S, Liu W, et al. A class III histidine kinase acts as a novel virulence factor in Botrytis cinerea. Mol Plant-Microbe Interact 2006;19:1042-50.

89. Yoshimi A, Kojima K, Takano Y, Tanaka C. Group III histidine kinase is a positive regulator of Hog1-type mitogen-activated protein kinase in filamentous fungi. Eukaryot Cell 2005;4:1820-8.

90. Lin $\mathrm{CH}$, Chung KR. Specialized and shared functions of the histidine kinaseand HOG1 MAP kinase-mediated signaling pathways in Alternaria alternata, the filamentous fungal pathogen of citrus. Fungal Genet Biol 2010;47:818-27.

91. Furukawa K, Hoshi Y, Maeda T, et al. Aspergillus nidulans HOG pathway is activated only by two-component signaling pathway in response to osmotic stress. Mol Microbiol 2005;56:1246-61.

92. Ferrigno P, Posas F, Koepp D, et al. Regulated nucleo/cytoplasmic exchange of HOG1 MAPK requires the importin $\beta$ homologs NMD5 and XP01. EMBO J 1998;17:5606-14.

93. Chauhan N, Latge JP, Calderone R. Signalling and oxidant adaptation in Candida albicans and Aspergillus fumigatus. Nat Rev Microbiol 2006;4:435-44.

94. Harding HP, Zhang Y, Zeng H, et al. 2003.
An integrated stress response regulates amino acid metabolism and resistance to oxidative stress. Mol Cell 2003;11:619-33.

95. Ikner A, Shiozaki K. Yeast signaling pathways in the oxidative stress response. Mutation Res 2005;569:13-27.

96. Kunkel BN, Brooks DM. Cross talk between signaling pathways in pathogen defense. Curr Opin Plant Biol 2002;5:325-31.

97. Martindale JL, Holbrook NJ. Cellular response to oxidative stress: signaling for suicide and survival. J Cell. Physiol 2002;192:1-15.

98. Pitzschke A, Forzani C, Hirt H. Reactive oxygen species signaling in plants. Antioxidants Redox Signal 2006;8:175764 .

99. Vranová E, Inzé D, Van Breusegem F. Signal transduction during oxidative stress. J Experment Bot 2002;53:1227-36.

100. He XJ, Mulford KE, Fassler JS. Oxidative stress function of the Saccharomyces cerevisiae Skn7 receiver domain. Eukaryot Cell 2009;8:768-78.

101. Morgan BA, Banks GR, Toone WM, et al. The Skn7 response regulator controls gene expression in the oxidative stress response of the budding yeast Saccharomyces cerevisiae. EMBO J 1997;16:1035-44.

102. Lu JM, Deschenes RJ, Fassler JS. Saccharomyces cerevisiae histidine phosphotransferase Ypdlp shuttles between the nucleus and cytoplasm for SLN1dependent phosphorylation of Ssk1p and Skn7p. Eukaryot Cell 2003;2:1304-14.

103. Lu JM, Deschenes RJ, Fassler JS. Role for the Ran binding protein, Moglp, in Saccharomyces cerevisiae signal transduction. Eukaryot. Cell 2004;3:1544-56.

104. He XJ, Fassler JS. Identification of novel Yaplp and Skn7p binding sites involved in the oxidative stress response of Saccharomyces cerevisiae. Mol Microbiol 2005;58:1454-67.

105. Wormley FLJr, Heinrich G, Miller J, et al. Identification and characterization of an SKN7 homologue in Cryptococcus neoformans. Infect Immun 2005;73:5022-30.

106. Fassler JS, West AH. Fungal Skn7 stress responses and their relationship to virulence. Eukaryot Cell 2011;10:156-67.

107. Lee J, Godon C, Lagniel G, et al. Yapl and Skn7 control two specialized oxidative stress response regulons in yeast. J Biol Chem 1999;274:16040-6.

108. Alberts AS, Bouquin N, Johnston LH, Treisman R. Analysis of RhoA-binding proteins reveals an interaction domain conserved in heterotrimeric G Protein b subunits and the yeast response regulator protein Skn7. J Biol Chem 1998;273:861622.

109. Bouquin N, Johnson AL, Morgan BA,
Johnston LH. Association of the cell cycle transcription factor Mbpl with the Skn7 response regulator in budding yeast. Mol Biol Cell 1999;10:3389-400.

110. Raitt DC, Johnson AL, Erkine AM, et al. The Skn7 response regulator of Saccharomyces cerevisiae interacts with Hsf1 in vivo and is required for the induction of heat shock genes by oxidative stress. Mol Biol Cell 2000;11:2335-47.

111. Williams KE, Cyer MS. The eukaryotic response regulator $\mathrm{Skn} 7 \mathrm{p}$ regulates calcineurin signaling through stabilization of Crzlp. EMBO J 2001;20:3473-83.

112. Brown JL, North S, Bussey H. SKN7, a yeast multicopy suppressor of a mutation affecting cell wall 1-glucan assembly, encodes a product with domains homologous to prokaryotic two-component regulators and to heat shock transcription factors. J Bacteriol 1993;175:6908-15.

113. Brown JL, Bussey H, Stewart RC. Yeast Skn7p functions in a eukaryotic two-component regulatory pathway. EMBO J 1994;13:5186-94.

114. Singh P, Neeraj Chauhan N, Ghosh A, et al. SKN7 of Candida albicans: mutant construction and phenotype analysis. Infect Immun 2004;72:2390-4.

115. Lamarre C, Ibrahim-Granet O, Du C, et al. Characterization of the SKN7 ortholog of Aspergillus fumigatus. Fungal Genet Biol 2007;44:682-90.

116. Vargas-Pérez I, Sanchez 0, Kawasaki L, et al. Response regulators SrrA and SskA are central components of a phosphorelay system involved in stress signal transduction and asexual sporulation in Aspergillus nidulans. Eukaryot Cell 2007;6:1570-83.

117. Izumitsu K, Yoshimi A, Tanaka C. Twocomponent response regulators Ssklp and Skn7p additively regulate high-osmolarity adaption and fungicide sensitivity in Cochliobolus heterostrophus. Eukaryot Cell 2007;6:171-81.

118. Saijo T, Miyazaki T, Izumikawa K, et al. Skn7p is involved in oxidative stress response and virulence of Candida glabrata. Mycopathologia 2010;169:81-90.

119. Hagiwara D, Mizuno T, Abe K. Characterization of the conserved phosphorylation site in the Aspergillus nidulans response regulator SrrA. Curr Genet 2011;57:103-14.

120. Nakamichi N, Yanada H, Aiba H, et al. Characterization of the Prrl response regulator with special reference to sexual development in Schizosaccharomyces pombe. Biosci Biotechnol Biochem 2003;67:547-55. 